\title{
EMOCIONES QUE INTERVIENEN EN EL APRENDIZAJE SIGNIFICATIVO DURANTE LOS PROCESOS DE ENSEÑANZA Y DE APRENDIZAJE
}

\section{Emotions involved in meaningful learning during teaching and learning processes}

https://doi.org/10.47606/ACVEN/PH0084

\author{
Zamira Cervantes Gil ${ }^{*}$ \\ https://orcid.org/0000-0003-1781-2964
}

Recibido: 12 febrero 2021 /Aprobado: 30 septiembre 2021

\section{RESUMEN}

El presente artículo tiene como objetivo reflexionar sobre los fundamentos teóricos del estudio de las emociones que intervienen en el aprendizaje significativo. Indagar en los fundamentos teóricos, implica hacer un recorrido en otras décadas acerca de las emociones en el proceso de enseñanza aprendizaje. Se presenta la información en cuatro apartados, que pretenden brindar un acercamiento y generar una reflexión sobre el estudio. Finalmente, conocer y trabajar a partir de la educación emocional puede contribuir, no sólo a desarrollar la investigación sobre las emociones en el campo educativo, sino también podría ayudar a las instituciones a desarrollar programas para apoyar a los profesores en la difícil tarea de educar, destacando el quehacer docente. De esta manera, reconocer las emociones en los procesos de enseñanza y de aprendizaje es potenciar la promoción de aprendizajes significativos dentro del aula.

Palabras clave: emociones, aprendizaje significativo, proceso de enseñanza y aprendizaje

1. Universidad Simón Bolívar, Barranquilla, Colombia

* Autor de correspondencia: zamiracervantesgil@gmail.com 


\section{ABSTRACT}

This article aims to reflect on the theoretical foundations of the study of emotions involved in meaningful learning. Investigating the theoretical foundations implies taking a journey in other decades about emotions in the teaching-learning process. The information is presented in four sections, which are intended to provide an approach and generate reflection on the study. Finally, knowing and working from emotional education can contribute, not only to developing research on emotions in the educational field, but could also help institutions to develop programs to support teachers in the difficult task of educating, highlighting the teaching task. In this way, recognizing emotions in the teaching and learning processes is to promote the promotion of meaningful learning within the classroom.

Keywords: emotions, meaningful learning, teaching and learning process

\section{Introducción}

En los últimos veinte años, las investigaciones en el área de la enseñanza señalan la importancia del estudio de las emociones en el proceso de enseñanza aprendizaje, como un proceso que forma parte de la construcción del conocimiento y puede ser una estrategia para conseguir nuevos conocimientos. Lo que supone que el interés por el estudio de las emociones en el terreno de la educación no es nuevo. En este orden de ideas, este estudio considera importante la mención de autores como Goleman (1998), Bisquerra (2000), Ibáñez (2002), Vivas (2003), Damasio (2005), Barragán et al. (2007), Rebollo et al. (2008), Albornoz, (2009), quienes consideraron en décadas pasadas la importancia de estudiar las emociones en el campo educativo. Para ello, el aprendizaje significativo resulta ser atractivo en la interacción entre el docente y el estudiante, por lo que no se puede ignorar la teoría planteada por Ausubel en 1963, la cual en la actualidad es interpretada y complementada por autores contemporáneos que han despertado su interés por estudiar las emocionesen el campo de la educación.

El presente estudio tiene como objetivo reflexionar acerca de los fundamentos teóricos del estudio de las emociones que intervienen en el aprendizaje significativo. Indagar en los fundamentos teóricos, implica hacer un recorrido en otras décadas sobre el reconocimiento de las emociones en el proceso de enseñanza aprendizaje, lo cual contribuye a plantearse las siguientes preguntas: ¿Cuáles son los fundamentos teóricos del aprendizaje significativo y del estudio de las emociones? ¿De qué manera las emociones intervienen en el proceso de enseñanza aprendizaje? ¿Cómo el quehacer docente puede promover el aprendizaje 
significativo? ¿Cuál es la importancia del estudio de las emociones en el campo educativo?

Así pues, este artículo propende brindar un acercamiento a los fundamentos teóricos de las emociones en el aprendizaje significativo, para generar una reflexión sobre la educación emocional que interviene en los procesos de enseñanza aprendizaje. Se presenta la información en cuatro apartados, que dan respuesta a las preguntas iniciales.

\section{Desarrollo}

El estudio científico de las emociones desde una perspectiva educativa ha estado marcado por la miopía de la ciencia del siglo XX, al tratar las emociones como objeto de estudio (Barragán et al. 2007). Un número creciente de investigaciones en las décadas pasadas, se centran en la regulación emocional (Rebollo et al. 2008). Es decir, las emociones tienen un papel preponderante en el aprendizaje. Asimismo, trabajar en torno a las dificultades que el estudiante percibe como relacionadas con su desarrollo personal y académico es permear el aprendizaje y estimular la motivación, la responsabilidad y el compromiso (Albornoz, 2009). Este proceso creador permitiría la transformación de la enseñanza, lo cual implica el reconocimiento de las emociones para adoptar una postura que contribuya a enfrentar el reto de aprender. A continuación, se presenta el desarrollo de las preguntas iniciales en cuatro apartados sobre el tema.

\section{Fundamentos teóricos del aprendizaje significativo y del estudio de las emociones}

El acto de enseñar y aprender está mediado por diferentes representaciones de un mismo conocimiento: la del profesor, la del estudiante y la del material didáctico (Ausubel, 1976). Así, el aprendizaje, en esta dinámica corresponde a un proceso continuo, personal, intencional, activo, dinámico, recursivo y de interacción (Agra et al. 2019). Siendo una estrategia prometedora en la enseñanza formal, según Ausubel (1963), autor de la Teoría del Aprendizaje Significativo (TAS); consiste en la interacción no arbitraria y no literal de nuevos conocimientos con conocimientos previos relevantes.

El punto central de la reflexión de Ausubel, es que de todos los factores que influyen en el aprendizaje, el más importante es lo que el estudiante sabe previamente (Moreira, 2016). Por su parte, Agra et al. (2019), proporcionan una definición actual del aprendizaje significativo, como la existencia de conocimientos previos que permiten la conexión con los nuevos conocimientos; interacción de los conocimientos previos y nuevos en la estructura cognitiva; ampliación de la estructura cognitiva a través de la incorporación de los conocimientos previos con los nuevos. 
En ese sentido, desentrañar lo que el estudiante ya sabe es más importante que identificar sus representaciones, conceptos e ideas, pues requiere considerar la totalidad del ser cultural y social en sus manifestaciones y lenguajes corporales, afectivos y cognitivos (Moreira, 2016). Según el autor, el profesor debe estar abierto para que el estudiante pueda revelar sus expectativas vividas, los objetos incorporados a su vida, las condiciones existenciales y no sólo el aspecto intelectual; es decir, no solo es importante el aspecto cognitivo del estudiante.

Al respecto, en el contexto interaccional en el aula (la interacción profesorestudiante/estudiante-estudiante), se constituye un modo de relación que tiene importantes consecuencias para la formación de los estudiantes. De esta manera, la percepción que tienen los estudiantes de sus relaciones interpersonales con los profesores sería lo que mayor impacto tiene en ellos, y no el contenido o materia de estudio (Guista, 2013). En consecuencia, las investigaciones examinan esta interacción, para identificar las emociones presentes en la consecución del aprendizaje significativo (Kamiloğlu, 2020). Los estudios realizados hasta el momento, trazan una perspectiva del aprendizaje que incluye la consideración de las características personales del estudiante, incluyendo los aspectos emocionales, los sentimientos, los patrones de comportamiento y el entorno (Guista, 2013).

Por su parte Albornoz (2009), cree que potenciar la expresión emocional dentro de la interacción en el aula, complementaría el desarrollo cognitivo, porque fijaría cualidades como la motivación o la responsabilidad hacia el acto de aprender. Además, según Gupta (2019), esto sugiere una priorización fundamental de las emociones en el sistema cognitivo-motivacional del estudiante. Del mismo modo, García (2012), plantea que las emociones implican una resignificación de los eventos o sucesos, lo que permite comprender y aceptar que la cognición y la emoción se afectan recíprocamente, por lo que la persona que se educa debe ser considerada en su razón y emoción. Lo anterior permite reafirmar que la razón y la emoción son dos aspectos que existen de manera conjunta y, porque se hayan inextricablemente unidos en la mente del individuo, lo que los lleva a actuar de manera conjunta, ligados a los conocimientos adquiridos.

De ahí que las emociones y sentimientos son importantes para el proceso de enseñanzaaprendizaje (Rodríguez, 2016), puesto que es necesario mantener una conducta motivada en los estudiantes para garantizar un aprendizaje significativo. Así mismo, Spunt y Adolphs (2019), señalan que estudiar los estados emocionales de las personas es una tarea difícil y compleja. No obstante, clasifican las emociones en categorías aprendidas culturalmente. Es decir, lo que se conoce como miedo, odio, felicidad, etc., depende del ambiente del sujeto. Es importante mencionar que a las emociones se les conoce como manifestaciones de los estados afectivos del ser humano (Rodríguez, 2016). 
Por lo anterior, en las últimas décadas, los investigadores empezaron a prestar atención al estudio de las emociones positivas en el campo educativo, ya que éstas tienen un significado esencial en los procesos de enseñanza y de aprendizaje (Gupta, 2019), lo que resulta atractivo en el reconocimiento de las emociones que intervienen en este proceso. Cabe destacar, que, la distinción entre emociones positivas y negativas es fundamental en el estudio de las emociones (Aviezer et al. 2012).

\section{Las emociones que intervienen en el proceso de enseñanza aprendizaje}

Al respecto, Ibáñez (2002), considera que, las emociones constituyen el aspecto de mayor relevancia para facilitar los aprendizajes en educación: según el autor, emociones positivas o gratas permitirán la realización de acciones favorables para el aprendizaje, emociones negativas o no gratas no lo permitirán. En el caso de la interacción en el aula, las emociones que fundan las acciones de los estudiantes serían determinantes para el curso que sigue su aprendizaje, al favorecer o limitar acciones de una cierta clase según sea la emoción que las sustente (García, 2012).

En un principio, Goleman (1998), al igual que muchos otros autores propone familias básicas de emociones, sin embargo, Damasio (2005), define las emociones primarias universales: sorpresa, miedo, felicidad, asco, tristeza, enojo. $\mathrm{Y}$ las clasifica:

- Primarias: miedo, rabia, alegría tristeza, disgusto.

- De fondo. Las emociones de fondo son esencialmente dos: entusiasmo y desánimo, son aquellas que constituyen nuestro estado de ánimo de fondo a lo largo del día y sobre las que construimos nuestras actuaciones conscientes.

- Sociales (también conocidas como emociones secundarias): vergüenza, desprecio, orgullo, envidia. Se denominan así porque requieren la presencia de una segunda persona para expresarse.

Este autor, establece la diferencia entre emociones y sentimientos. La idea esencial que extrae Damasio (2018), es que los sentimientos son experiencias mentales de estados corporales, que surgen cuando el cerebro interpreta emociones, que son estados físicos que surgen de las respuestas del cuerpo a los estímulos externos. En ese sentido, Rodríguez (2016), considera que los aprendizajes significativos son producto de la interacción que fluye entre el docente y el estudiante permeados por emociones, los cuales internalizados en un proceso entre la razón y la emoción garantiza un efecto deseado.

De la misma manera, es importante señalar que los procesos emocionales deben ser considerados en el campo educativo, puesto que estos no solamente ocurren en el interior del sujeto, sino que también son parte de la interacción social 
(Machorro-Cabello y Valdez, 2019). Circunstancia por la que suelen llamarse procesos psicosociales, los cuales conforman la base central de la educación. Lo expuesto anteriormente indica que la tarea del educador es importante en este estudio.

\section{El quehacer docente que interviene en el aprendizaje significativo}

Considerando el tema de las emociones en educación, Bisquerra (2012), menciona cómo la educación emocional puede ayudar a gestionar adecuadamente las emociones de los estudiantes. Lo anterior conlleva mencionar la tarea del docente, el cual, según el autor, debe desarrollar habilidades que le ayuden a educar emocionalmente.

Lo señalado anteriormente, destaca el quehacer del docente al promover el aprendizaje significativo de los estudiantes considerando la relevancia de las emociones en los actores del proceso de enseñanza y de aprendizaje, dado que las emociones del estudiante y del docente se ponen en práctica durante cada clase. Por lo tanto, si el docente promueve emociones positivas como el amor, la felicidad, entonces se generará un ambiente propicio. Pero si el docente genera emociones negativas como el miedo, la ansiedad, la ira, la hostilidad estará generando un ambiente poco propicio para promover el aprendizaje significativo (García, 2012), entendido éste, como aquél que se promueve cuando los contenidos están relacionados con lo que el estudiante ya sabe, la relación no ha de ser arbitraria, como lo señala Ausubel (Díaz, 2010).

Esto hace énfasis en la necesidad de la educación emocional por parte de los docentes (Machorro-Cabello y Valdez, 2019). Lo que supone la necesidad que requiere una reflexión profunda acerca de la importancia de brindar educación emocional a los estudiantes para generar emociones positivas que les motiven a: aprender, conocerse, identificar sus capacidades y habilidades, confiar en ellos, respetar a las personas y el medio ambiente. Es decir, el docente debe constituirse en un mediador esencial de las habilidades emocionales en el estudiante (MachorroCabello y Valdez, 2019).

\section{Importancia del estudio de las emociones en el campo educativo}

Es importante destacar que, la preocupación por la incorporación de las habilidades sociales y emocionales en la educación no es nueva. Autores como Vivas (2003), mencionan la educación emocional, como un proceso de desarrollo humano, que abarca tanto lo personal como lo social e implica cambios en las estructuras cognitiva, actitudinal y procedimental (Bisquerra, 2000). Por lo tanto, reconocer la importancia del estudio de las emociones en el campo educativo, es destacar la educación emocional como un proceso continuo que debe estar presente a lo largo de todo el currículum académico. 
La educación emocional debe tener un carácter participativo porque requiere de la acción conjunta y cooperativa de todos los que integran la estructura académicodocente-administrativa de las instituciones educativas y porque es un proceso que exige la participación individual y la interacción social (Vivas, 2003). Así mismo, la educación emocional debe ser flexible en cuanto debe estar sujeta a un proceso de revisión y evaluación permanente que permita su adaptabilidad a las necesidades de los estudiantes (Machorro-Cabello y Valdez, 2019).Además, se destaca el papel fundamental que juegan los docentes en el desarrollo de la capacidad emocional de sus estudiantes, por lo que su propio desarrollo emocional, así como su preparación en este campo resulta una responsabilidad ineludible de los centros de formación docente.

\section{Reflexión}

En el campo educativo, tal como se ha venido planteando se puede trabajar con los estudiantes para que respondan emocionalmente, es decir, se puede generar conciencia sobre las emociones presentes durante las clases, lo cual responsabiliza al docente, promoviendo nuevas actitudes en los estudiantes, con el fin de hacerlos más receptivos a la hora de adquirir nuevos conocimientos, propiciando de esta manera un estadio consciente de lo que los motiva a aprender, generando en ellos aprendizajes significativos.

Por tanto, es relevante considerar lo planteado por Según Rodríguez (2016), los estados emocionales representan un hecho significativo para el campo educativo, puesto que a partir del dominio y manejo de las emociones se pueden transformar las prácticas de enseñanza y aprendizaje de una manera significativa. Por lo tanto, el docente se ha constituido en un mediador esencial de las habilidades emocionales en el estudiante (Rodríguez, 2016); debe seleccionar, programar y presentar al estudiante aquellos estímulos que modifiquen su trayectoria emocional, le hagan sentirse bien consigo mismo y desarrolle su capacidad para regular sus reacciones emocionales al momento de obtener un aprendizaje significativo (Vivas, 2003).

Por su parte, en la necesidad de reconocer la importancia de las emociones en el aprendizaje significativo, el Decreto colombiano 2230 de 2003, por el cual se modifica la estructura del Ministerio de Educación Nacional, y se dictan otras disposiciones, se reconoce la importancia de lograr la formación del educando en términos moral, espiritual, intelectual, física y afectiva. Desde esa perspectiva, las emociones constituyen un factor importante al momento de interpretar el comportamiento humano, influyendo fuertemente para regular su actividad y su conducta, puesto que dependiendo del tipo de emoción en que se encuentre el ser humano será el tipo de acción que pueda realizar (Rodríguez, 2016). 
Ahora bien, la ley 115 de 1994, por la cual se expide la ley general de la educación, en el artículo quinto, menciona la importancia que tiene la adquisición y generación de los conocimientos científicos y técnicos, mediante la apropiación de hábitos intelectuales adecuados para el desarrollo del saber, lo que implica la participación del proceso de enseñanza aprendizaje. Por lo tanto, para generar espacios de aprendizaje, y lograr aprendizajes significativos, es indispensable hacer una reestructuración de metodologías didácticas y currículos, enfocados a una educación más personalizada, donde el educando sea un sujeto activo y participativo de su propio aprendizaje (Astroza, 2019) y, sea tenida en cuenta su individualidad. En ese sentido, la consecución de aprendizajes significativos, debe generar cambios en las metodologías y la didáctica. Así, las emociones representan junto a los procesos cognitivos un factor determinante en la adquisición del conocimiento (Rodríguez, 2016), por lo cual, se empezaría a trabajar por experiencias de aprendizaje agradables, que generen emociones que propicien aprendizajes significativos. Por lo tanto, en este proceso, es fundamental el rol docente.

\section{Conclusiones}

Considerando los aspectos señalados, se considera importante destacar las emociones presentes en el proceso de enseñanza y aprendizaje para la consecución del aprendizaje significativo, puesto que en este proceso las emociones juegan un papel importante en la motivación del estudiante, lo cual destaca el rol del docente para lograrlo. Asumiendo, que la interacción en el aula, sigue siendo importante para obtener aprendizajes.

Conocer y trabajar a partir de la educación emocional puede contribuir no sólo a desarrollar la investigación sobre el estudio de las emociones en el campo educativo, sino también podría ayudar a las instituciones a desarrollar programas para apoyar a los profesores en la difícil tarea de educar.En consecuencia, en el campo educativo, tal como se ha venido planteando se puede estimular a los estudiantes partiendo del reconocimiento del rol docente. Finalmente, reconocer las emociones en los procesos de enseñanza aprendizaje es potenciar la promoción de aprendizajes significativos dentro del aula.

\section{REFERENCIAS}

Agra, G., Formiga, NS., Oliveira, PS., Costa, MML.,Fernandes, MDGM, Nóbrega, MMLD. (2019). Analysis of the concept of Meaningful Learning in light of the Ausubel's Theory. Rev Bras Enferm;72(1):248-255. English, Portuguese. doi: 10.1590/0034-7167-2017-0691. https://pubmed.ncbi.nlm.nih.gov/30916292/ 
Albornoz, Y. (2009). Emoción, música y aprendizaje significativo. Educere; 13(44):67-73. Recuperado de: http://ve.scielo.org/scielo.php?script=sci_arttext\&pid=S131649102009000100008

Ausubel, D. (1963). The psychology of meaningful verbal learning. New York: Grune\&Stratton.

Ausubel, D. (1976). Psicología educativa. Un punto de vista cognoscitivo. México: Ed. Trillas.

Astroza, J. (2019). Procesos para potenciar el aprendizaje significativo y favorecer la dimensión cognitiva por medio de ambientes de aprendizaje de los niños y niñas de 4 a 5 años de la Institución Educativa "el jazmín" del barrio primavera en la ciudad de Bogotá. Universidad Santo Tomás

Aviezer, H., Trope, Y., y Todorov, A. (2012). Body cues, not facial expressions, discriminate between intense positive and negative emotions. Science (New York, N.Y.); 338(6111):1225-1229. https://doi.org/10.1126/science.1224313

Barragán, R., Buzón, O., y García, R. (2007). Diagnóstico y evaluación de aprendizajes universitarios con eportafolios: regulación de aprendizajes Blended-Learning y nuevos roles del alumnado. Actas del XIII Congreso Nacional de Modelos de Investigación Educativa, AIDIPE: San Sebastián.

Bisquerra, R. (2000). Educación emocional y bienestar. Barcelona: Praxis.

Bisquerra, R. (2012). Orientación, tutoría y educación emocional. España. Síntesis.

Damasio, A. (2018). The Strange Order of Things. Nueva York: Penguin Books.

Damasio, A. (2005). En busca de Spinza. Neurobiología de la emoción y los sentimientos. Barcelona:46 - 52 .

Decreto 2230 de Agosto 8 de 2003. Recuperado de: https://www.mineducacion.gov.co/1621/articles-86029_archivo_pdf.pdf

Díaz, F. (2010). Estrategias docentes para un aprendizaje significativo. Una interpretación constructivista. México, Mc Graw -Hill

García, J. (2012). La educación emocional, su importancia en el proceso de aprendizaje. Revista Educación;36(1):1-24. Recuperado de: https://www.redalyc.org/pdf/440/44023984007.pdf

Gupta R. (2019). Positive emotions have a unique capacity to capture attention. Progress in brain research; 247:23-46. https://doi.org/10.1016/bs.pbr.2019.02.001 
Guista, A. (2013). Concepções de aprendizagem e práticas pedagógicas. EducRev,29(1):20-36. doi: 10.1590/S0102-46982013000100003

Goleman, D. (1998). La práctica de la inteligencia Emocional. Editorial Kairos. Barcelona

Ibáñez, N. (2002). Las emociones en el aula. Estudios pedagógicos (Valdivia); (28):31-45. Recuperado de: https://scielo.conicyt.cl/scielo.php?pid=S071807052002000100002\&script=sci_ arttext\&tlng=p\#0

Kamiloğlu, R., Fischer, A., y Sauter, D. (2020). Good vibrations: A review of vocal expressions of positive emotions. Psychonomic bulletin \& review, 27(2):237265. https://doi.org/10.3758/s13423-019-01701-x

Ley 115 de 1994.(3 de julio de 2021). Secretaría Senado. Recuperado de: http://www.secretariasenado.gov.co/senado/basedoc/ley_0115_1994.html

Machorro-Cabello, M., y Valdez, V. (2019). La educación emocional como factor para potenciar el aprendizaje significativo. Vida Científica Boletín Científico De La Escuela Preparatoria, 4, 7(14): 18-22. Recuperado de: https://repository.uaeh.edu.mx/revistas/index.php/prepa4/article/view/4180

Moreira, M. (2016). Enseñanza de la física: aprendizaje significativo, aprendizaje mecánico y criticida de. Rev Ensenanza de la Física, 26(1):45-52. Recuperado de:

http://www.revistas.unc.edu.ar/index.php/revistaEF/article/viewFile/9515/10290

Rebollo, M., García, R., Barragán, R., Buzón, O., y Vega, L. (2008). Las emociones en el aprendizaje online. Relieve;14 (1):1-23. Recuperado de: https://core.ac.uk/download/pdf/71040072.pdf

Rodríguez, Y. (2016). Las emociones en el proceso de enseñanza-aprendizaje. Vinculando.

Recuperado

de:

https://vinculando.org/psicologia_psicoterapia/emociones-proceso-ensenanzaaprendizaje.html?utm_source=feedburner\&utm_medium=email\&utm_campaig $\mathrm{n}=$ Feed\%3A+Vinculando+(Revista+Vinculando)\&format=pdf\#vcite

Spunt, R., y Adolphs, R. (2019). The neuroscience of understanding the emotions of others. Neuroscienceletters:

693:44-48. https://doi.org/10.1016/j.neulet.2017.06.018

Vivas, M. (2003). La educación emocional: conceptos fundamentales. Sapiens. Revista Universitaria de Investigación:4(2). Recuperado de: https://www.redalyc.org/pdf/410/41040202.pdf 